\title{
Advances in Experiments and Modeling in Micro- and Nano-Biomechanics: A Mini Review
}

\author{
Mian Long, ${ }^{1}$ Masaaki Sato, ${ }^{2}$ Chwee Teck Lim, ${ }^{3}$ Jianhua Wu, ${ }^{4}$ Taiji Adachi, ${ }^{5}$ and Yasuhiro Inoue ${ }^{5}$ \\ ${ }^{1}$ Key Laboratory of Microgravity, Center of Biomechanics and Bioengineering, Institute of Mechanics, Chinese Academy of \\ Sciences, Beijing 100190, China; ${ }^{2}$ Graduate School of Biomedical Engineering, Tohoku University, Sendai 980-8579, Japan; \\ ${ }^{3}$ Division of Bioengineering, Department of Mechanical Engineering, Mechanobiology Institute, National University of \\ Singapore, Singapore 117576, Singapore; ${ }^{4}$ Institute of Biomechanics, School of Bioscience and Bioengineering, South China \\ University of Technology, Guangzhou, China; and ${ }^{5}$ Institute for Frontier Medical Sciences, Kyoto University, Kyoto, Japan
}

(Received 2 April 2011; accepted 3 June 2011; published online 15 June 2011)

Associate Editor Edward Guo oversaw the review of this article.

\begin{abstract}
Recent advances in micro- and nano-technologies and high-end computing have enabled the development of new experimental and modeling approaches to study biomechanics at the micro- and nano-scales that were previously not possible. These new cutting-edge approaches are contributing toward our understanding in emerging areas such as mechanobiology and mechanochemistry. Another important potential contribution lies in translational medicine, since biomechanical studies at the cellular and molecular levels have direct relevance in areas such disease diagnosis, nano-medicine and drug delivery. Thus, the developed experimental and modeling approaches are critical in elucidating important mechanistic insights in both basic sciences and clinical treatment. While it is hard to cover all the recent advances in this mini-review, we focus on several important approaches. For experimental techniques, we review the assays involving shear flow, cellular imaging, microbead, microcontact printing, and micropillars at the micro-scale, and micropipette aspiration, optical tweezers, parallel flow chamber, and atomic force microscopy at the nano-scale. In modeling and simulations, we outline the theoretical modeling for actin dynamics in migrating cell and actin-based cell motility in cellular mechanics, as well as the receptor-ligand binding in cell adhesion and the application of free, steered, and flow molecular dynamics simulations in molecular biomechanics. Relevant scientific issues and applications are also discussed.
\end{abstract}

Keywords-Cellular mechanics, Molecular biomechanics, Experimental techniques, Theoretical modeling, Computations.

Address correspondence to Mian Long, Key Laboratory of Microgravity, Center of Biomechanics and Bioengineering, Institute of Mechanics, Chinese Academy of Sciences, Beijing 100190, China; Masaaki Sato, Graduate School of Biomedical Engineering, Tohoku University, Sendai 980-8579, Japan; Chwee Teck Lim, Division of Bioengineering, Department of Mechanical Engineering, Mechanobiology Institute, National University of Singapore, Singapore 117576, Singapore. Electronic mails: mlong@imech.ac.cn, sato@bml.mech. tohoku.ac.jp, ctlim@nus.edu.sg

\section{INTRODUCTION}

Cells are constantly subjected to and regulated by both chemical and physical factors in its microenvironment. In particular, these physical factors include mechanical forces as well as topography and elasticity of the extracellular matrix. As such, studying biomechanics at the cellular and molecular levels is important to our understanding of how such physical or mechanical factors regulate cell functions in both health and disease. With the recent advances in micro- and nano-technologies as well as high-end computing, we are observing a burgeoning of new experimental and computational approaches to study the biomechanics of biological systems at the microand nano-scales. These new and cutting-edge approaches are also fostering a stronger integration between the disciplines of biomechanics and modern biology (cell and molecular biology, genomics, proteomics and systems biology), and allows engineers, physicists, chemists, and biologists to collectively address fundamental issues at the cellular and molecular levels. The knowledge acquired and the cutting-edge technologies developed are also helpful in translating the discoveries in this basic biomechanical study into useful applications in molecular and cell engineering and even in developing novel approaches to diagnose and treat diseases. This mini-review summarizes some of the recent advances in experimental and computational approaches in the field of cellular and molecular mechanics and discusses how they can contribute toward addressing important issues in mechanobiology and mechanochemistry.

Considering the fact that thousands of bioengineers have put tremendous efforts into these areas in the past century, it is very challenging to cover all the 
fundamental and important advancements in the minireview. Here we focus on four aspects: (1) cellular biomechanics techniques including shear flow assay, cellular imaging, and microfabricated technologies for cell mechanics, (2) modeling and simulation of subcellular mechanics of cell migration and motility, (3) molecular biomechanics techniques including micropipette aspiration, optical tweezers, parallel flow chamber, and atomic force microscopy, and (4) molecular dynamics simulation and mathematical modeling of individual molecule or molecular complex. We hope to showcase the latest contributions not only from around the world, but also from that of the Asian community.

\section{CELL MECHANICS AND MECHANOBIOLOGY}

In general, cell mechanics refers to how the elastic and adhesive properties of cells are changed and/or regulated under various physiological and pathological conditions, while mechanobiology refers to how mechanical cues (e.g., shear flow, geometrical patterns, substrate topography and elasticity, etc) elicit various biological responses and functions.

\section{Experimental Techniques}

\section{Shear Flow Assay}

Fluid flow is one of the important environmental components that a cell may be subjected to. Examples include endothelial and smooth muscle cells in blood and lymph vessels, osteoblasts and osteoclasts cells in bone, and epithelial cells in esophagus and intestines. These cells are continually exposed to flow of blood, lymph, tissue fluid, and digested food among others, in which the physical forces exerted do evoke or regulate cell functions. Fluid flow can be divided into two major types: laminar and turbulent. Various types of devices have been developed to apply fluid flow to cultured cells in vitro, depending on their different objectives. There have been many reports focusing on vascular endothelial cells (ECs) exposed to fluid flow or fluid shear stress (FSS). Hemodynamic FSS acting on vascular ECs evokes a variety of cellular responses, including proliferation, ${ }^{53}$ expression of adhesive molecules ${ }^{70}$ cytoskeletal structures and morphology, ${ }^{29}$ and mechanical properties, ${ }^{84}$ that may be relevant to both the physiology and pathology of blood vessels. Many previous studies have attempted to determine the mechanisms by which ECs sense FSS and adapt to such mechanical factors. Here we summarize the devices and image analysis being employed in these studies.
The major devices for subjecting cultured cells to laminar flow are the rotating disk type and parallelplate flow chamber type.

In the rotating disk devices, there are the paralleldisk $^{74}$ and cone-plate ${ }^{22}$ types. Shear stress, $\tau$, to be applied to cells in the parallel plate type is expressed as follows, $\tau=\mu r \omega / h$, where $\mu$ is the viscosity of fluid, $r$ the distance from the axis of rotation, $\omega$ the angular velocity, and $h$ the distance between the two parallel plates. Characteristics of this device are values of shear stress that are dependent upon the distance from the rotational axis. In the case of the cone-plate type, since the distance $h$ between the two surfaces of cone and plate is a function of $r$ and $\alpha$ (angle between the cone and the plate) where $h=r \tan \alpha$, shear stress is constant regardless of position and can be expressed as, $\tau=\mu \omega / \tan \alpha$. The rotating devices are usually used to attain unsteady or turbulent flow by applying high rotating speed and cone angle. ${ }^{17}$

The parallel-plate flow chamber ${ }^{27,42,52}$ is commonly used to apply shear stress to cultured cells. Shear stress exerted on the cell surface can be expressed by the equation, $\tau=6 Q \mu / b h^{2}$, where $Q$ is flow rate and $b$ and $h$ are the width and height of the flow channel, respectively. Various types of flow chamber have been designed to allow for a wide range of shear stress by changing the width ${ }^{50,95}$ and height. ${ }^{65}$ In addition, the effects of disturbed flow can also be examined in the parallel-plate flow chamber by adding a step in the channel. ${ }^{16,21,71}$ Some experiments were focused on examining the impact of spatial gradient of shear stress within the chamber. To obtain the high spatial gradient, $T$-shaped flow chamber was developed and utilized from the viewpoint of genesis and growth of cerebral aneurysm..$^{82,89}$

\section{Cellular Imaging}

Recently, FSS-induced activation of several candidates of mechanosensitive molecules, such as G proteins $^{83}$ and PECAM, ${ }^{85}$ has been demonstrated. However, there is no primary evidence as to whether these molecules are activated directly by mechanical loading or by intracellular signaling interactions that were prompted by another mechanosensor. This comes from the difficulties in precisely describing the intracellular mechanical conditions, i.e., how FSS acting on the apical surface of ECs is transmitted and generates an intracellular mechanical field. As such, it is necessary to investigate the degree to which forces are exerted on the intercellular junction, focal adhesion, and other candidates for mechanotransducers.

In an attempt to solve this problem, Ueki et al. ${ }^{93}$ developed a novel experimental technique that enables the direct observation of the passive deformation of 
living ECs exposed to the physiological range of FSS by confocal microscopy and the measurement of the intracellular strain field together with the application of image processing and the finite element method (FEM) ${ }^{18}$ They stained cytoplasmic domain and nuclei of living ECs with fluorescent dyes, respectively. The cells were cultured in parallel-plate flow chamber and the flow rate was controlled by a syringe pump. The scanning line of laser microscope was set to be parallel to the direction of flow crossing the vicinity of the center of the nucleus and containing two to four ECs in one frame. A personal computer with a customized program was used to synchronize the pushing motion of the syringe pump and the trigger signal for image acquisition by the microscope for a temporally wellcoordinated measurement. To obtain the intracellular displacement field, an image correlation analysis was carried out between the undeformed and deformed images. The obtained lateral images of endothelial cells exposed to shear stress and the strain distribution in the cell calculated from the displacement field by FEM are shown in Fig. 1. The dynamic image during deformation under shear stress exposure can be found at the journal homepage: www.elsevier.com/locate/ ybbrc.

Another technique is FRET (fluorescence resonance energy transfer) imaging to visualize the spatiotemporal activations of signaling proteins in a cell such as Rho family GTPase. Various kinds of FRET probes have been developed. For example, Raichu (Ras and interacting chimeric unit)-Racl and - RhoA are typical to visualize Racl and RhoA in living cells. ${ }^{72,73}$

\section{Microbead Techniques}

Although it remains unclear which molecule or cellular component is the primary mechanosensor, one of the major candidates is focal adhesions (FAs) consisting of integrins and associated molecules. Since FAs provide the mechanical linkage between the extracellular matrix and cytoskeletons, forces would be directly exerted on FAs when cells are exposed to external forces. Recent reports have shown that p130CAS ${ }^{85}$ and $\operatorname{talin}^{18}$ in FAs serve as primary mechanosensors, which trigger signal transduction cascades leading to alteration in cellular functions such as proliferation ${ }^{86}$ and gene expression. ${ }^{28}$ In addition, FAs may adapt to their mechanical environment by changing their structures and connections to cytoskeletons, and have an important role in mechanicallyinduced morphological and cytoskeletal remodeling of ECs. Previous studies using micro-manipulation techniques reported the accumulation of FA-associated molecules ${ }^{30}$ and $\operatorname{actin}^{20,38}$ when forces were applied to FAs, and local stiffening of cells ${ }^{63}$ was induced by continuous or cyclic forces.

One of the typical methods of applying localized mechanical force is the use of microbeads. Glass and magnetic microbeads coated with a protein or a polypeptide are used to adhere to FAs. ${ }^{34,94,99}$ Wang and $\mathrm{Suo}^{99}$ reported that stress fibers have a role in force transmission. They applied mechanical stress at the apical side using magnetic bead technique, and then observed deformation at the basal side under low and high prestress, the tension acting in the cell. They
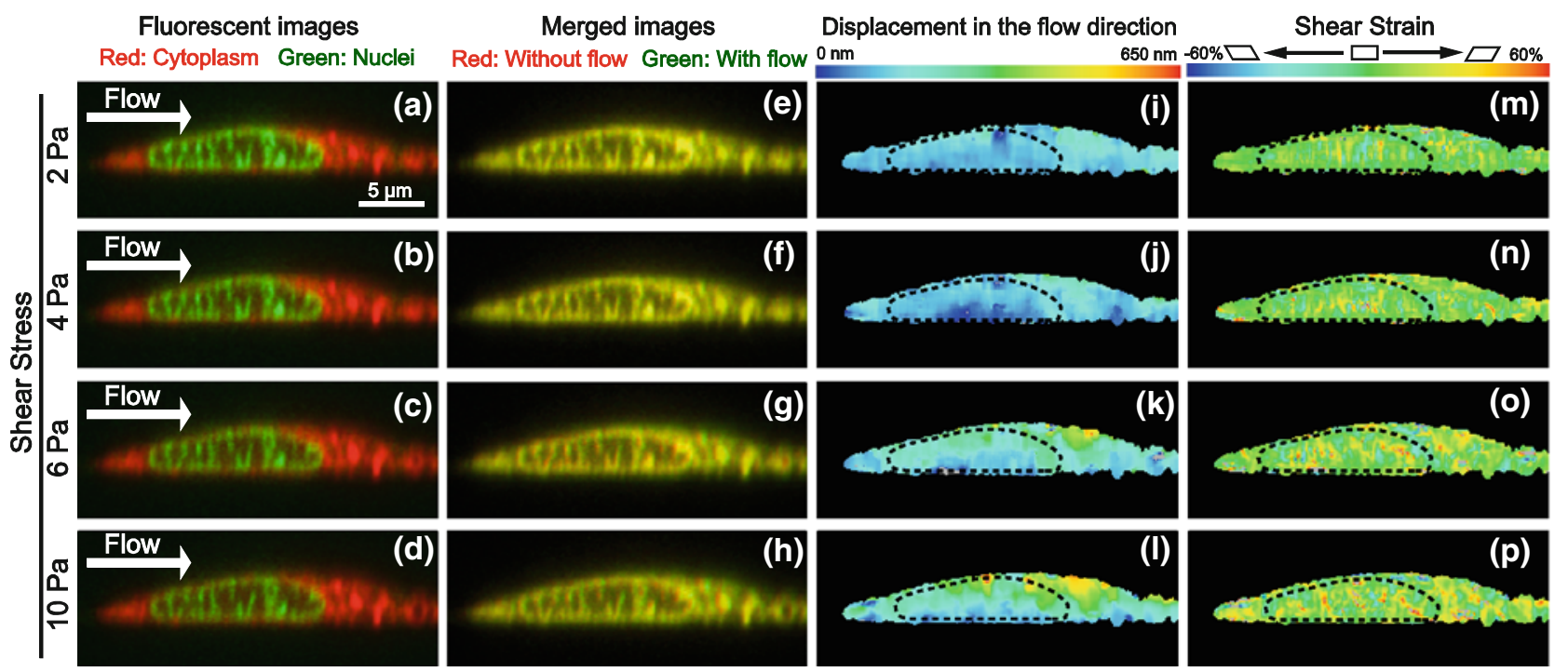

FIGURE 1. Lateral images of HUVECs exposed to FSSs of 2 (a, e, i, m), 4 (b, f, j, n), 6 (c, g, k, o), and 10 Pa (d, h, I, p). (a-d) Fluorescent images of nuclei (green) and cytoplasm (red) of HUVECs under given FSS. (e-h) Merged images of HUVECs under static (red) and flow (green) conditions. Disagreement of green and red colors indicates the displacement due to the flow. (i-l) Contour mappings of displacement in the flow direction. $(m-p)$ Contour mappings of shear strain analyzed using FEM. The dextral and sinistral shear strains are indicated by warm and cold colors, respectively. 
obtained basal deformation map at low prestress which led to localized displacement. However, at high prestress, mechanical stress was transmitted over a long distance. Another example is shown by Hayakawa et $a l .{ }^{34}$ by introducing a glass microbead to the cell surface. Stress fibers were developed to connect the apical FAs to the basal ones. When they moved the bead with glass micropipette by $4 \mu \mathrm{m}$, they observed the movement of small fluorescent beads with diameter of $50 \mathrm{~nm}$ embedded in the substrate gel. However, only very small displacement of beads was observed in cytochalasin-D treated cells. This observation has further supported that mechanical force transmits from the apical FAs to the basal ones.

\section{Microtechnologies for Cell Mechanics}

Recently, some developed micro-fabricated technologies have greatly enabled the study of cell mechanics and mechanobiology and in elucidating how they can regulate important biological processes such as cell adhesion, migration, spreading, and differentiation.

\section{Microcontact Printing}

Microcontact printing provides a simple way of producing patterns on substrates for altering and regulating cell shape, spreading and migration. One example is the use of microcontact printing to produce fibronectin patterns of varying sizes to demonstrate that the shape and spreading area of human mesenchymal stem cells (hMSCs) can regulate the differentiation of stem cells into specific lineages. Here, hMSCs seeded on small patterns of fibronectin (about
$1024 \mu \mathrm{m}^{2}$ ) tended to differentiate into adipocytes (fat cells) while those seeded on large fibronectin patterns (about $10,000 \mu \mathrm{m}^{2}$ ) tended to differentiate into osteocytes (bone cells) ${ }^{64}$ Other examples include the use of various other microcontact printed geometrical patterns (e.g., rectangle with different aspect ratios or pentagons with varying curvature of the edges) to show that the patterns that induce the increase of intracellular actomyosin tension regulate the differentiation behavior of MSCs. ${ }^{44}$

Microcontact printed pattern of fibronectin is also another simple way of regulating cell shape and influencing the cell division axis (Fig. 2). This axis along which a cell divides determines the position and fate of the daughter cells. In fact, the spatial distribution of extracellular matrix (ECM) was found to determine cortical actin dynamics which in turn regulate the axis of cell division. ${ }^{91,92}$ Hence, it is important to understand how biomechanical cues imposed by the ECM proteins regulate cell division.

Cell migration is known to be influenced not only by chemical but also mechanical cues presented in their microenvironment including topography, geometrical constraints, and ECM protein distribution patterns. Micro-fabrication techniques have enabled us to systematically alter these mechanical cues to study how these factors can regulate cell migration. One example is the use of micro-patterned substrates to demonstrate that the migration of cells on very narrow patterns (one-dimensional or 1D migration) is faster than that on two-dimensional or 2D surfaces. A contributing reason is that the migration on narrow channels is much more dependent on myosin II than that of $2 \mathrm{D}$ migration. $^{23}$
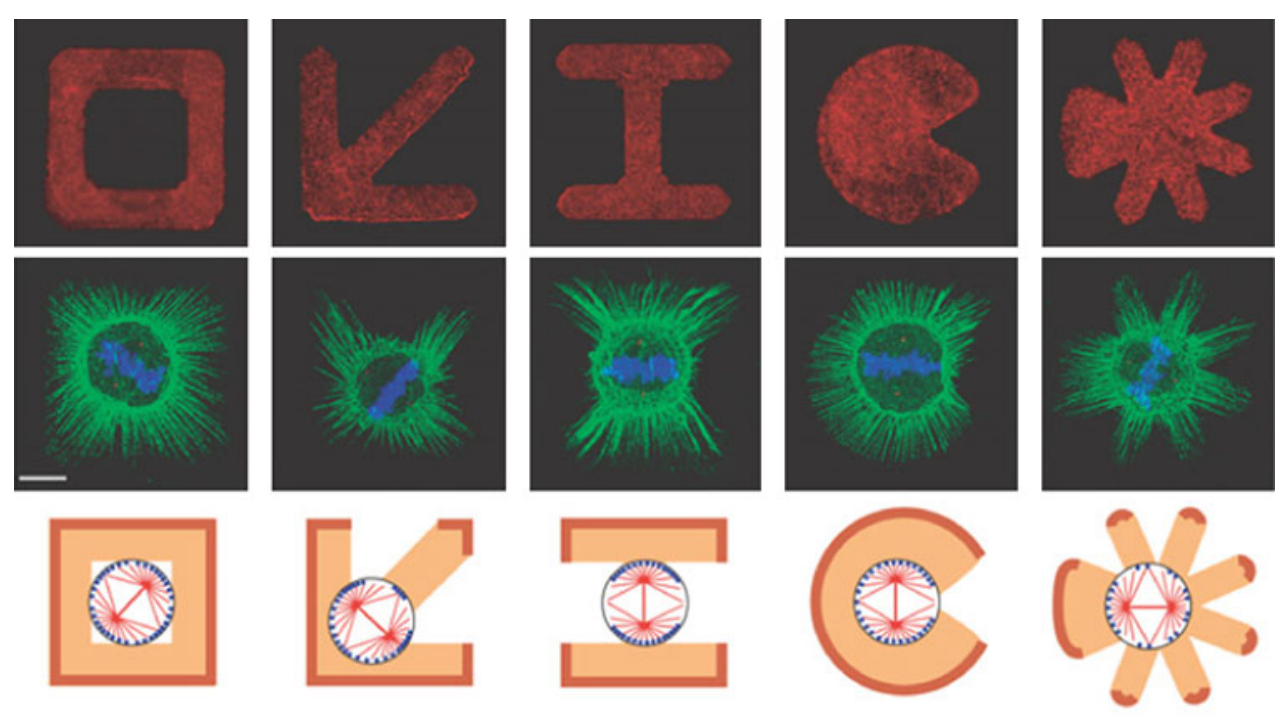

FIGURE 2. Demonstration of how various ECM patterns regulate the cell division axis. ${ }^{91}$ [Reprinted by permission from Macmillan Publishers Ltd: Nature, 447:493-496, copyright 2007]. 

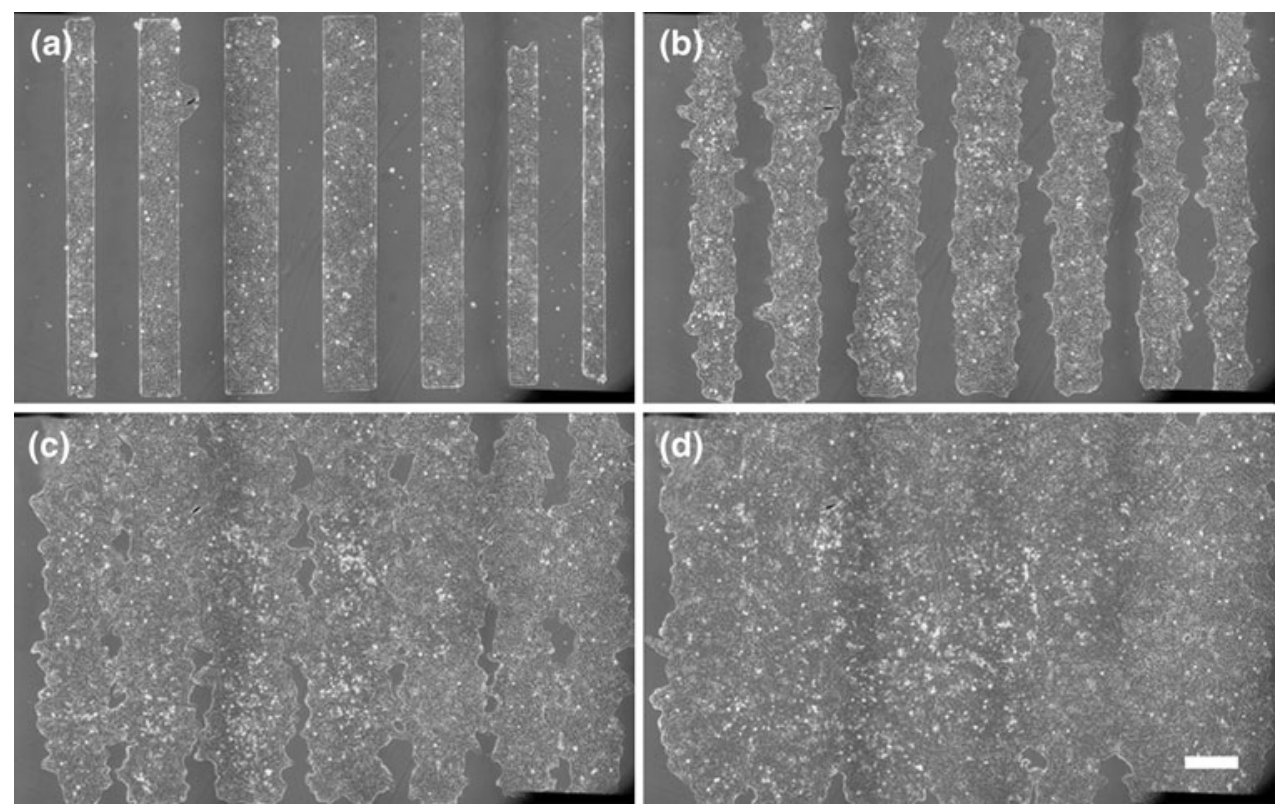

FIGURE 3. A microstencil based method to produce a multiple models of wounds for collective cell migration study ${ }^{80}$ [Copyright 2007 National Academy of Sciences, USA].

Collective cell migration is relevant to several important biological processes such as wound healing and cancer metastasis. Recently, a micro-fabricated soft elastic "microstencil" was used to study wound healing by first placing it on a cell culture surface. ${ }^{80}$ Once cells had grown to confluence within the stencil, the stencil was then lifted off which resulted in multiple injury-free wounds for cell migration observation (Fig. 3). Here, cell migration behaviors were characterized as both collective and individualistic. While many cells moved in a collective and coordinated way, there were some very active "leader cells" fingering the borders and acting in a very fibroblast-like and nonepithelial manner.

\section{Micropillar Assays}

The micropillars are typically made of PDMS and the corresponding assays consist of arrays of pillars whose stiffness is controlled by their diameter, height, and curing conditions. Also, the tips of these micropillars are coated with ECM proteins to allow for effective attachment and migration of cells on top of these pillars.

Micropillared substrate is often used to characterize cellular traction forces by observing the extent to which the cells deflect the micropillars. They have been used to measure traction forces exerted by epithelial cells sheets ${ }^{24}$ as well as single migrating cells. Other examples include the use of micropillar substrate to probe the chemo-mechanical effects of anti-cancer drugs such as emodin on cancer cells. ${ }^{102}$

Apart from measuring cellular traction forces, micropillar substrates have also been used for

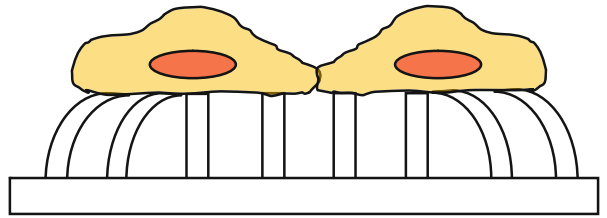

FIGURE 4. Two cells on fibronectin stamped micropillar substrate form an intercellular adhesion. The force of intercellular adhesion is equal and opposite to the sum of the forces exerted by each single cell on the micropillars.

characterizing the intercellular adhesion forces between cells. Here, micropillars were first microcontact printed with a "bowtie" pattern of fibronectin. ${ }^{56}$ Cells were subsequently allowed to adhere and spread on these patterns and the cell substrate traction forces were determined from the deflection of the micropillars (Fig. 4). Using this technique it was shown that mechanical tugging force regulated the size of intercellular adhesion.

\section{Modeling and Simulations}

\section{Actin Dynamics in Migrating Cell: Molecular-Scale Modeling Approaches}

Cell migration is a crucial process for many physiological and pathological events, such as embryogenesis and cancer metastasis. During cell migration, a lamellipodium is formed, which is a flat and broad membrane extension filled with a dense and highly branched actin network. ${ }^{87,96}$ Protrusion of the lamellipodial leading edge, referred to as membrane protrusion, 
is one of the essential cellular activities for continuous cell migration because it brings the front margin of the migrating cell forward. ${ }^{51}$ During membrane protrusion, branched actin filaments are polarized with their barbed ends in the migrating direction and polymerized beneath the membrane. The chemical energy gain from the polymerization is consumed by mechanical work of the membrane protrusion. ${ }^{8,79}$

To explain the mechanochemical aspect of force generation that drives membrane protrusion, a mathematical model has been constructed: the Brownian ratchet (BR). ${ }^{67,77}$ According to the $\mathrm{BR}$, the membrane in front of the actin filament is thermally fluctuated in its position. The membrane fluctuations create a sufficient gap for monomers to intercalate and to polymerize at the barbed end. Thus, the polymerized actin filaments beneath the membrane rectify the Brownian motion of the membrane so as to produce a unidirectional force. Subsequent modeling extends the BR to flexible actin filaments: the elastic BR. If the actin filament is long, its own thermal undulations can also create a gap between its barbed end and the membrane. ${ }^{68}$ By applying the BR models, the protrusion velocity of not only lamellipodia ${ }^{66,67}$ but also filopo$\mathrm{dia}^{3,69}$ has been analyzed successfully. The fundamental understanding of these molecular machineries has attracted new interests regarding their relationships with cellular behaviors over several spatial and temporal scales.

Non-muscle myosin II and actin constitute the major force-generating machinery of actomyosin networks, where actomyosin contractility is fundamental to cellular reshaping and movement. ${ }^{97}$ Therefore, these mechanical behaviors of actomyosin networks are recognized as being fundamental to biological functions, ${ }^{54}$ but the mechanochemical basis of the emergence of these functions is still unclear.

At the individual actin filament level, several mathematical models of actin networks, such as semi-flexible polymers without cross-links ${ }^{90}$ and with cross-links ${ }^{37,45}$ have been proposed. These models have successfully simulated the dynamics of actin networks and clarified its relationship with rheological properties, whereas processive myosin movements which is the origin of the contractility of actomyosin networks, has not been considered. On the other hand, at the cellular-scale, the cable network model (CNM) has been suggested for studying contractile actin networks, which has demonstrated that the mechanics of a contractile filamentous network with a spatial distribution of adhesions is important in determining cellular shape. ${ }^{75}$ Because the $\mathrm{CNM}$ relies on a lattice discretization of the mean field of the actin cytoskeleton, it illustrates the computational efficiency for cellular-scale simulations. However, the cables described in the CNM are not meant to represent individual actin filaments. Therefore, analyzing the dynamic rearrangement of actomyosin networks is beyond the scope of the CNM. Gathering and extending these mathematical models at the individual filament level, it gives challenging opportunities for better understanding of force generation and dynamic rearrangement in actomyosin networks.

\section{Actin-Based Cell Motility: Cellular-Scale Modeling Approaches}

Recently, several experiments have been performed to relate actin dynamics in migrating cell with cellularscale activities such as the shapes of migrating cells. ${ }^{43,49,104}$ To enhance the knowledge of these multiscale relationships, theoretical and computational studies at the whole-cell level are needed.

Several studies to construct whole-cell models and simulate cell migration involved with various cell shapes have been reported. Rubinstein et al. have proposed the $2 \mathrm{D}$ model of the fish keratocyte. ${ }^{81}$ This model incorporates lamellipodial protrusion, cell adhesion, contraction and actin transport. The simulations using the model reproduce observed cell shapes, forces, and movements qualitatively, and give an explanation about some experimental results of perturbations on the actin machinery. For example, it has been observed experimentally that photoreleasing a caged thymosin at one side of a keratocytes lamellipodium induces a pivotal motion of the keratocytes around the perturbed side. The model has successfully explained this cellular response to the perturbation from the view point of $\mathrm{G}$-actin concentration. Maree et al. have suggested a 2D whole-cell model of the fish keratocyte based on the cellular potts model that stochastically determines the leading edge protrusion where force-velocity relation in the protrusion is fitted to the thermal ratchet force-velocity curve in the model. ${ }^{62}$ In addition, their model takes into account the effects of some chemical signal transductions such as $\mathrm{Cdc} 42$, Rac, and Rho. The model gives an insight into how the keratocyte can maintain its shape and polarity and how it can alter direction in response to changes in its environment in terms of interactions of Cdc42, Rac and Rho.

However, these models are phenomenological and not derived on the basis of the underlying molecular mechanism that has been considered by the BR. Although the cellular-scale simulations using the BR with a membrane load has been analyzed, ${ }^{31,66}$ it is unable to be used for studies in which resultant shapes of the cells are unknown. This is because the BR requires a priori constant load that depends on the cell shape.

Recently, Inoue and Adachi have suggested a 2D keratocyte model based on the coarse-grained BR that 


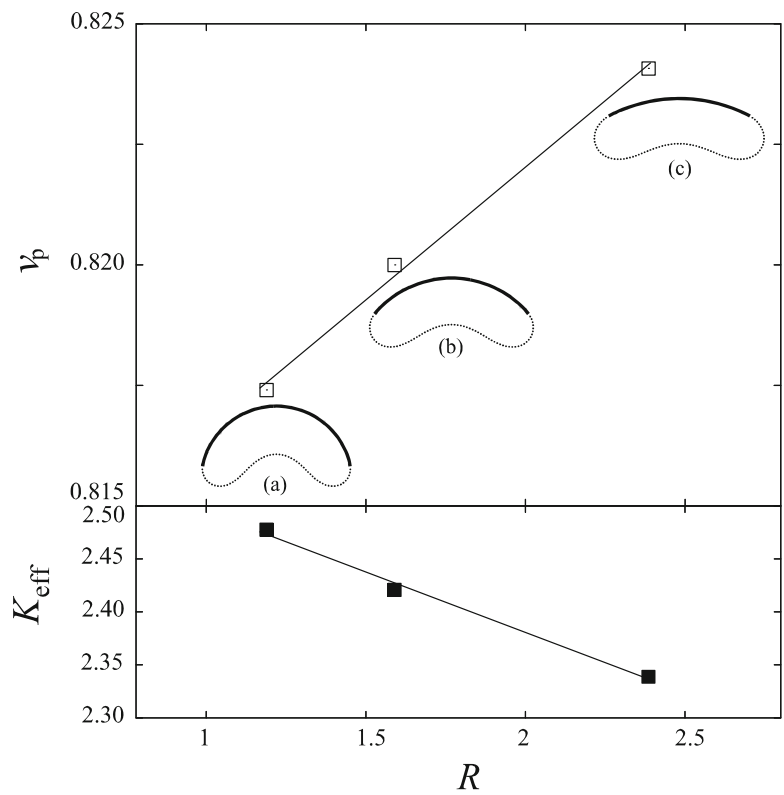

FIGURE 5. Protrusion velocity, $v_{\mathrm{p}}$, and effective elastic constant of the leading edge, $K_{\text {eff }}$, measured in $10^{6} \mathrm{k}_{\mathrm{B}} \mathrm{T} / \mu \mathrm{m}^{2}$ as a function of the radius of the curvature of the leading edge, $R$ $(\mu \mathrm{m})$. The radius is obtained by a least square fitting of the equation of a circle to a set of positional vectors of nodes allocated to the leading edge. Open and solid squares indicate the values of $v_{\mathrm{p}}$ and $K_{\mathrm{eff}}$, respectively. All lines are only for the sake of reference. Simulated shapes of the leading edge are indicated by the solid line, wherein a critical stall velocity is 0.80 (a), 0.81 (b), and 0.82 (c).

is derived using non-equilibrium thermodynamics theory on the basis of the underlying molecular mechanism. ${ }^{39}$ Because they assume that the cell is either stationary or steady migrating, the cell adhesion and contraction can be simply expressed in terms of an energy constraint at the cellular level. This model estimates the protrusion velocity, $v_{\mathrm{p}}$, consistently with an effective elastic constant, $K_{\text {eff }}$, which represents the state of the energy of the membrane, and reproduces the experimentally-observed keratocyte shapes by the simulation (Fig. 5). The trend of dependences of the protrusion velocity on the curvature of the leading edge, the temperature, and the substrate stiffness has also agreed with the other experimental results.

While these mathematical modeling of cell migrations have achieved significant success, they are still 2D models. Thus, further efforts should now focus on investigating $3 \mathrm{D}$ environments.

\section{MOLECULAR BIOMECHANICS AND MECHANOCHEMISTRY}

Molecular biomechanics focuses on how a single molecule behaves mechanically and/or how two counterpart molecules interact with each other, while mechanochemistry refers to how mechanical stimuli (enforced contact, restrained fluctuation, shear flow, etc) regulate the conformational changes of biomolecules and affect their biochemical reactions and biological functions.

\section{Experimental Techniques}

\section{Micropipette Aspiration}

Micropipette aspiration technique (MAT) was first used in quantifying the mechanical and viscoelastic properties of an isolated cell in the mid-1950s when a suction pressure was applied on the cell via a pipette. In the past two decades, MAT has been widely employed to understand the binding kinetics and rupture force of surface-bound receptor-ligand interactions using two experimental protocols: the adhesion frequency assay (left panel) and the biomembrane force probe (BFP) assay (right panel) (Fig. 6a). Data analysis is conducted on the measured data to predict the kinetic rate/binding affinity and rupture force/bond lifetime. In the adhesion frequency assay, the adhesion frequency, defined as the fraction of adhesive events over the total number of tests conducted and measured over the systematically-varied contact duration and site densities of receptors and ligands, are predicted using a probabilistic model of small system kinetics ${ }^{15}$ to collect the forward and reverse rates and binding affinity. ${ }^{35,101}$ In the biomembrane force probe assay, the rupture force, defined as the product of membrane deflection and membrane stiffness, is measured on the systematically varied loading rate. Dynamic force spectroscopy (DFS) theorem that defines the correlation of rupture force to loading rate $^{25}$ is then used to predict the parameters of energy landscape upon Bell model. ${ }^{4}$ Bond lifetime, defined as the time interval during which the bond remains bound, is measured on the systematically-varied applied force. A first-order dissociation kinetics model is then used to predict the reverse rate or bond lifetime.

MAT and BFP assays have been widely applied to test various adhesive molecular pairs, such as $\mathrm{Fc} \gamma \mathrm{R}$ IgG,${ }^{15}$ selectin-ligand, ${ }^{35,101}$ integrin-ligand, ${ }^{107}$ and TCR-MHCII binding. ${ }^{36}$ While at least one type of molecule of interest needs to be purified from the cell membrane and re-captured onto a RBC or bead as a force transducer in these conventional assays, a gasdriven MAT newly-developed is able to determine directly the binding kinetics of interacting molecules constitutively expressed on nucleated cells (left panel in Fig. 6a).

\section{Optical Tweezers Manipulation}

Optical tweezers (OT) assay was first applied by trapping the biological particles in the late $1980 \mathrm{s.}^{2} \mathrm{An}$ 


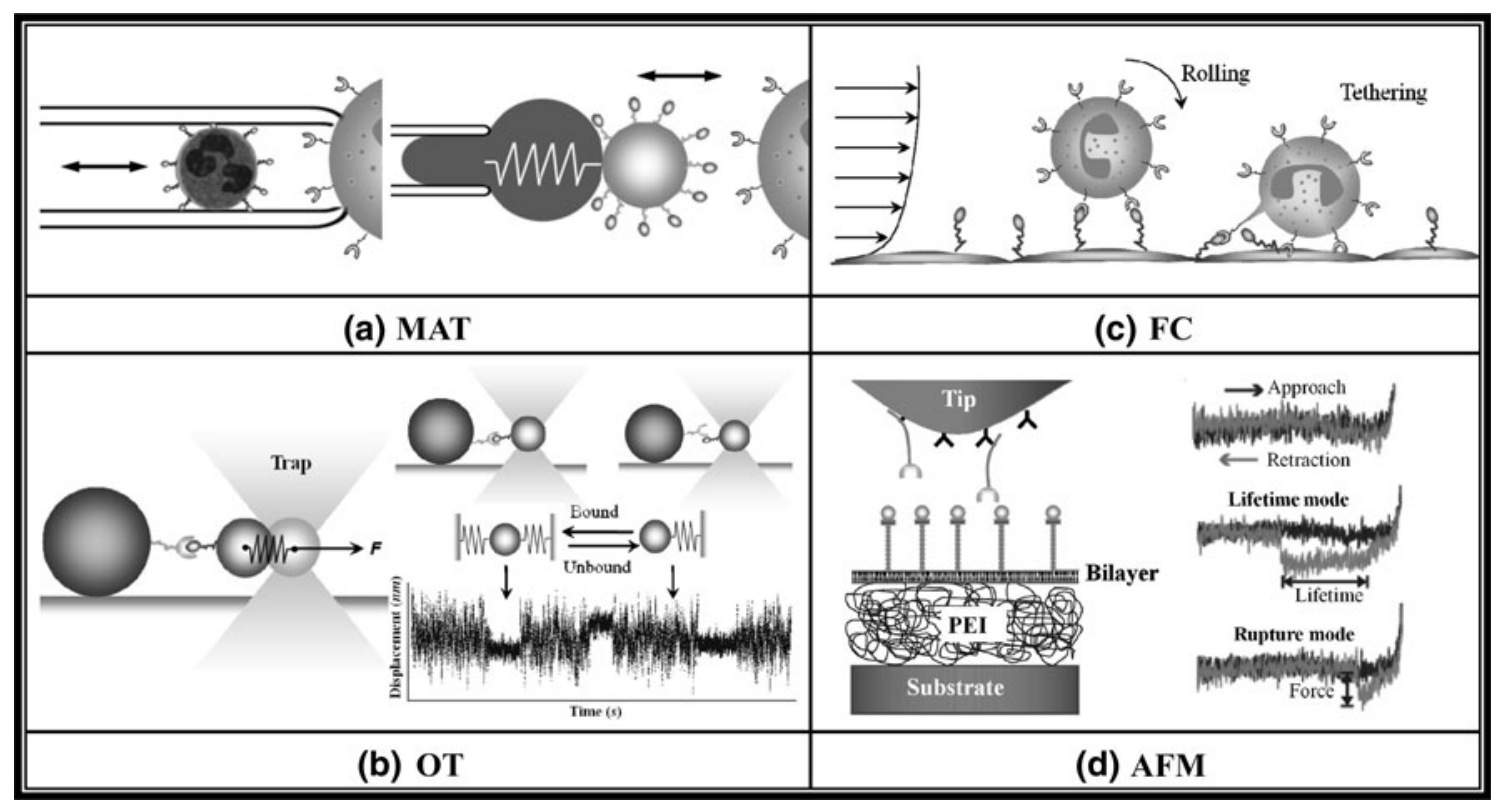

FIGURE 6. Schematics of MAT (a), OT (b), FC (c), and AFM (d) assays. (a) A gas driven MAT assay (left) and a BFP assay (right). (b) An OT assay for monitoring bond rupture (left) and association kinetics (right). (c) A FC assay for neutrophils rolling (left) and tethering (right). (d) An AFM assay for system functioning (left) and working protocols (right).

adhesive event is identified when the bead/cell is bounced back to the trap center during withdrawal (left panel in Fig. 6b). The advantage of OT assay lies in its features of non-contact, low perturbation, and high spatial and temporal resolutions, which enables one to conduct the delicate, near-equilibrium tests for unbinding and association kinetics of molecular pair of interest. The protocol for unbinding kinetics is the same as that done in MAT assay. The other for association kinetics has been recently developed based upon the thermal fluctuation rationale (right panel in Fig. 6b) ${ }^{88}$ in which the time course of bead displacement is monitored for the occurrence of sequential binding and unbinding events, as is also observed in $\mathrm{BFP}^{11}$ assay. To date, OT assay has been widely applied in many molecular systems to understand the molecular biomechanics and biophysics at a highlysensitive resolution.

\section{Parallel Flow Chamber}

Parallel flow chamber (FC) technique, similar to that mentioned earlier for studying cell mechanics, has also been applied to quantify the molecular mechanics of cell rolling. ${ }^{41}$ A laminar flow is usually applied to drive a receptor-expressing cell/bead flowing over the ligand-immobilized substrate or surface. Time course of the binding of cell/bead is visualized when it encounters the substrate and various adhesion patterns are identified mainly as rolling (left panel) and (transient) tethering (right panel) (Fig. 6c) of the flowing cell/bead. Collected cell accumulation, detachment, tethering and rolling, and transient rolling, are then used to determine the kinetics of interacting molecules under systematically-varied shear stress and site density of interacting molecules. A first-order dissociation kinetics model that defines the irreversible unbinding is used to predict the bond lifetime at a given shear stress, together with Bell's model for the force dependence of bond lifetime.

FC serves as an in vitro assay to mimic the physiological flow in many biological processes such as inflammatory cascade, tumor metastasis, and thrombus formation. It has been widely used to understand the cell rolling over and tethering on the substrate mediated by receptor-ligand interactions under blood flow.

\section{Atomic Force Microscopy}

Atomic force microscopy (AFM) was first developed in the mid 1980s based upon the scanning tunnel microscope. ${ }^{7}$ An adhesive event is identified when the cantilever is deflected during withdrawal (left panel in Fig. 6d), and the measured data is employed to predict the rupture force/bond lifetime. Two modes are applied to quantify the mechanochemistry of receptorligand bond under applied force: the rupture force mode works at a given loading rate while the bond lifetime mode operates at a given applied force (right panel in Fig. 6d). Mechano-chemical coupling models that define the dependence of reverse rate on applied 
force, ${ }^{4,19}$ together with DFS theorem, ${ }^{25}$ are then used to predict the parameters of kinetic rates and energy landscape.

AFM assay has now been widely applied to understand the forced dissociation at the single molecule level. By combining with micro-fabricated technologies, it is also possible to build up an AFM array to probe the multiple events or species simultaneously.

\section{Simulations and Modeling}

\section{Molecular Dynamic Simulation of Molecular Biomechanics}

By incorporating both conformational changes and atomic details of biomolecules in a $3 \mathrm{D}$ environment with different temperatures, pressures, and/or mechanical constraints, molecular dynamics simulation (MDS) provides functional implication and yields information that is not possible through any other means. ${ }^{1}$ Today, MDS is routinely applied in investigating the respective mechano-chemical coupling and mechanical properties of biomolecules at the single molecular level. ${ }^{1}$ Variable simulation protocols and analysis methods such as free, ${ }^{59}$ steered, ${ }^{78}$ and flow molecular dynamics, ${ }^{59}$ have been developed to manifest the interesting properties of biomolecules.

Classical or free MDS has been widely used to probe conformational stability, flexibility, and folding/ unfolding pathway of proteins. For example, the instability of GPIb $\alpha \beta$-hairpin without vWF is demonstrated by a free MDS as a spontaneous transition to a structureless loop. ${ }^{59}$ But for vWF-A1/GPIb $\alpha$ complex, MDS exhibits its conformational flexibility and the stabilizing electrostatic interactions between these two proteins. ${ }^{40}$ The unfolding of the central $\beta$-sheet of vWF-A2 is proposed to start from its edges and then propagate into its center. ${ }^{13}$ The allostery of P-selectin lectin (Lec) domain followed by an epithelial growth factor (EGF)-like domain is recently visualized using free MDS. ${ }^{61}$ These simulations shed light on the question that what aspects, such as topology, hydrogen-bonding patterns, and core interactions, determine the mechanical properties of a protein. ${ }^{26}$

Compared with free MDS, a steered molecular dynamics (SMD) simulation is more popular in investigating mechano-chemical coupling and mechanical properties of biomolecules due to the inherent similarity to AFM, OT, and BFP assays as well as DFS experiments. In SMD, external forces are applied to molecules to probe their mechanical properties, as well as to accelerate processes that are otherwise too slow to model. ${ }^{78}$ As an in silico complement of single-molecule techniques, SMD simulations have been extensively used in many studies.
Lü and Long have applied SMD to stretch a single P-selectin construct and suggested that the burst of intramolecular hydrogen bonds is the main cause of the structural collapses. ${ }^{60}$ From simulation of unfolding of vWF A domains by tensile force, Chen et al. have observed two different unfolding pathways of $\beta$-strands, the sliding and unzipping pathways being encountered by higher and lower energy barriers, respectively. ${ }^{12}$ Liu et al. have estimated the elastic modulus of antimicrobial peptide $\operatorname{HP}(2-20)$ and its four analogues through SMD and further proposed a rigidity-enhanced antimicrobial activation of the peptides. ${ }^{55}$ SMD simulations on unbinding of receptor from its ligand, such as P-selectin glycoprotein ligand 1 (PSGL-1) from P-selectin and glycoprotein Ib $\alpha$ (GPI$\mathrm{b} \alpha$ ) from $v W F-A 1$, have provided insights into the molecular mechanism underlying catch-bond. ${ }^{32,40,58,103}$

Flow MDS was inspired from FC assay and first carried out by Lou and $\mathrm{Zhu}^{59}$ and further improved by Chen et $a l .{ }^{14}$ Upon flow MDS, the authors have observed the significant conformational change on the $\beta$-switch of GPIb $\alpha$ extracellular domain, and suggested a structural explanation of flow-enhanced affinity of GPIb $\alpha$ and vWF. ${ }^{59}$ Zou et al. have compared a coarselattice model and a freely jointed chain model so as to illustrate how the folding rate and conformational transition of $\beta$-hairpin depend on the entropic and enthalpic energies, the latter controlled by flow show. ${ }^{109}$ Wang and Sandberg ${ }^{98}$ have observed the unfolding of ubiquitin through flow MDS, in which flow is generated by pulling two frozen water surfaces along a given direction. These results have exhibited potential ability of flow MDS in modeling biological process under flow at the single molecular level.

The timescale of MD simulation generally yields several nanoseconds, but the experimental time window (or dynamical range) is about $10 \mathrm{~ms}, 1 \mathrm{~ms}$, or $10 \mu$ s for optical tweezers, BFP, or AFM techniques, respectively. This timescale gap of 3-6 magnitude orders between MDS and single biomolecular experiments has certainly limited the application of MD. Recently, several studies with individual trajectories longer than one microsecond have been reported. ${ }^{46}$ The gap is becoming narrower with the improvements in molecular dynamics algorithms, software, and computer hardware.

\section{Mathematical Modeling on Receptor-Ligand Binding in Cell Adhesion}

As a key step in many physiological processes, cell adhesion under flow is a mechanochemical coupling process, including initial tethering, rolling, and firm adhesion, and is mediated by receptor-ligand interaction. ${ }^{106}$ Attention has been paid to theoretical 
modeling on force-dependent receptor-ligand binding in cell adhesion, in order to better understand the events of cell adhesion and extract reaction kinetic information of adhesive molecules from measured data.

Bell model, the best-known theoretical model derived from thermodynamic analysis on specific adhesive events between the cells, demonstrates slipbond mechanism of receptor-ligand interaction under applied forces, ${ }^{4,5}$ but fails to describe catch-bond behavior which governs flow-enhanced cell adhesion. Two-pathway model and deformation model are developed to depict the transition from catch-bond to slip-bond in receptor-ligand interaction under forces. ${ }^{32}$ Force-dependent association models have provided a way to estimate reverse rate of receptor unbinding from its ligand in AFM, OT, and BFP assays as well as DFS measurements. To date, the force dependence of reverse rate of receptor-ligand interaction has been intensively studied by different methods, including lifetime and rupture force measurements of single bonds or tether and rupture force measurements of single cell. ${ }^{11}$

Cell adhesion mediated by receptor-ligand interaction refers to a $2 \mathrm{D}$ kinetic process at the cell contact area. Currently, it is still a challenge to relate molecular binding kinetics measured in $3 \mathrm{D}$ conditions to that in $2 \mathrm{D}$ or membrane-bound cases. Both probabilistic and deterministic models have been developed in the past decades. For example, Chesla et al. have developed a probabilistic model of molecular kinetics for a small system with a few bonds (most likely one) forming during a contact with short duration and low densities of both receptors and ligands on the apposing surfaces. ${ }^{15}$ By contrast, $\mathrm{Wu}$ et al. ${ }^{100}$ have used a deterministic description of force-free, coupled reaction-diffusion model to determine $2 \mathrm{D}$ kinetics of receptor-ligand interaction in a contact-area FRAP (fluorescence recovery after photobleaching) assay where sufficient bonds in contact area are required in stable cell adhesion. The association rate so measured from any one of above two assays is a lumped parameter with contact area, which is usually unknown in MAT, ${ }^{15}$ but can be roughly estimated in FRAP measurements. ${ }^{100}$

Modeling for cell adhesion mediated by receptorligand interactions under flow has long been attractive to investigators. In the adhesive dynamics model introduced by Hammer and Apte, ${ }^{33}$ the balance of forces and torques on a cell flowing near a surface due to hydrodynamic shear and ligand-receptor bonds has been considered for cell adhesion on vessel wall and the cell movements from free flowing, tethering, rolling motion to firm adhesion are well modeled. $., 9,10,47,48$ Moreover, Long et al. ${ }^{57}$ have developed a probabilistic model, instead of Monte Carlo simulation, to simulate the shear-induced formation and breakage of doublets cross-linked by receptor-ligand bonds for cell aggregation inside blood vessel. Cellular properties including microvilli tethering and cytoskeletal deformation are important in mediating cell rolling. ${ }^{105}$ To examine the dynamic contact forces on leukocyte microvilli, Zhao et al. have presented a theoretical model and predicted that contact force increases nonlinearly with shear and that only the longest microvilli contacts the substrate at high shear stress $>0.2 \mathrm{dyn} / \mathrm{cm}^{2} .{ }^{108} \mathrm{Yu}$ and Shao have further developed a model to understand the effect of cell membrane tether extraction on neutrophil rolling stabilization and indicated that simultaneous tether extraction from the neutrophil and endothelial cell increases bond lifetime, which has made more transient tethers to be stable and then let rolling neutrophils to be more shear-resistant. ${ }^{105}$ Pawar et al. have modeled cell body and microvillus deformation and predicted that both catch-slip-bond behavior and lesser cell deformation are responsible for threshold phenomenon observed on selectin-mediated leukocyte rolling over ligand-immobilized substrate under shear flow. ${ }^{76}$

\section{CONCLUDING REMARKS}

In this mini-review, we briefly reported the recent advances in the experimental and modeling aspects of micro- and nano-biomechanics research. These developments are helpful in enabling us to further understand the mechanisms involved in cell mechanics and mechanobiology as well as molecule biomechanics and mechanochemistry.

It is hoped that in the near future and with further progress made in this area, we will be able to better integrate the information obtained at the cell and molecular levels and provide a clearer insight into the mechanical transduction and signaling from cell to biomolecule and vice versa. Such findings will certainly be helpful in further elucidating the basic functions of biological systems at the cellular and molecular levels.

\section{ACKNOWLEDGMENT}

The authors are grateful for technical assistances from Dr. Yan Zhang.

\section{REFERENCES}

\footnotetext{
${ }^{1}$ Adcock, S. A., and J. A. McCammon. Molecular dynamics: survey of methods for simulating the activity of proteins. Chem. Rev. 106:1589-1615, 2006.
} 
${ }^{2}$ Ashkin, A., J. M. Dziedzic, and T. Yamane. Optical trapping and manipulation of single cells using infraredlaser beams. Nature 330:769-771, 1987.

${ }^{3}$ Atilgan, E., D. Wirtz, and S. X. Sun. Mechanics and dynamics of actin-driven thin membrane protrusions. Biophys. J. 90:65-76, 2006.

${ }^{4}$ Bell, G. I. Models for specific adhesion of cells to cells. Science 200:618-627, 1978.

${ }^{5}$ Bell, G. I., M. Dembo, and P. Bongrand. Cell adhesion. Competition between nonspecific repulsion and specific bonding. Biophys. J. 45:1051-1064, 1984.

${ }^{6}$ Bhatia, S. K., M. R. King, and D. A. Hammer. The state diagram for cell adhesion mediated by two receptors. Biophys. J. 84:2671-2690, 2003.

${ }^{7}$ Binnig, G., C. F. Quate, and C. Gerber. Atomic force microscope. Phys. Rev. Lett. 56:930-933, 1986.

${ }^{8}$ Borisy, G. G., and T. M. Svitkina. Actin machinery: pushing the envelope. Curr. Opin. Cell Biol. 12:104-112, 2000.

${ }^{9}$ Caputo, K. E., and D. A. Hammer. Adhesive dynamics simulation of G-protein-mediated chemokine-activated neutrophil adhesion. Biophys. J. 96:2989-3004, 2009.

${ }^{10}$ Chang, K. C., D. F. J. Tees, and D. A. Hammer. The state diagram for cell adhesion under flow: leukocyte rolling and firm adhesion. Proc. Natl Acad. Sci. USA 97:11262-11267, 2000.

${ }^{11}$ Chen, W., E. A. Evans, R. P. McEver, and C. Zhu. Monitoring receptor-ligand interactions between surfaces by thermal fluctuations. Biophys. J. 94:694-701, 2008.

${ }^{12}$ Chen, W., J. Z. Lou, and C. Zhu. Molecular dynamics simulated unfolding of von Willebrand factor A domains by force. Cell. Mol. Bioeng. 2:75-86, 2009.

${ }^{13}$ Chen, W., J. Z. Lou, and C. Zhu. Simulated thermal unfolding of the von Willebrand factor A domains. Cell. Mol. Bioeng. 3:117-127, 2010.

${ }^{14}$ Chen, Z., J. Z. Lou, C. Zhu, and K. Schulten. Flow-induced structural transition in the beta-switch region of glycoprotein Ib. Biophys. J. 95:1303-1313, 2008.

${ }^{15}$ Chesla, S. E., P. Selvaraj, and C. Zhu. Measuring twodimensional receptor-ligand binding kinetics by micropipette. Biophys. J. 75:1553-1572, 1998.

${ }^{16}$ Chiu, J. J., C. N. Chen, P. L. Lee, C. T. Yang, H. S. Chuang, et al. Analysis of the effect of disturbed flow on monocytic adhesion to endothelial cells. J. Biomech. 36:1883-1895, 2003.

${ }^{17}$ Davies, P. F., A. Remuzzi, E. J. Gordon, C. F. Dewey, Jr., and M. A. Gimbrone, Jr. Turbulent fluid shear stress induces vascular endothelial cell turnover in vitro. Proc. Natl Acad. Sci. USA 83:2114-2117, 1986.

${ }^{18}$ del Rio, A., R. Perez-Jimenez, R. C. Liu, P. Roca-Cusachs, J. M. Fernandez, et al. Stretching single talin rod molecules activates vinculin binding. Science 323:638-641, 2009.

${ }^{19}$ Dembo, M., D. C. Torney, K. Saxman, and D. Hammer. The reaction-limited kinetics of membrane-to-surface adhesion and detachment. Proc. R. Soc. Lond. B Biol. Sci. 234:55-83, 1988.

${ }^{20}$ Deng, L. H., N. J. Fairbank, B. Fabry, P. G. Smith, and G. N. Maksym. Localized mechanical stress induces timedependent actin cytoskeletal remodeling and stiffening in cultured airway smooth muscle cells. Am. J. Physiol. Cell Physiol. 287:C440-C448, 2004.

${ }^{21}$ DePaola, N., P. F. Davies, W. F. Pritchard, L. Florez, N. Harbeck, et al. Spatial and temporal regulation of gap junction connexin43 in vascular endothelial cells exposed to controlled disturbed flows in vitro. Proc. Natl Acad. Sci. USA 96:3154-3159, 1999.
${ }^{22}$ Dewey, C. F., S. R. Bussolari, M. A. Gimbrone, and P. F. Davies. The Dynamic response of vascular endothelialcells to fluid shear-stress. J. Biomech. Eng. 103:177-185, 1981.

${ }^{23}$ Doyle, A. D., F. W. Wang, K. Matsumoto, and K. M. Yamada. One-dimensional topography underlies threedimensional fibrillar cell migration. J. Cell Biol. 184:481490, 2009.

${ }^{24} \mathrm{du}$ Roure, O., A. Saez, A. Buguin, R. H. Austin, P. Chavrier, et al. Force mapping in epithelial cell migration. Proc. Natl Acad. Sci. USA 102:2390-2395, 2005.

${ }^{25}$ Evans, E., K. Ritchie, and R. Merkel. Sensitive force technique to probe molecular adhesion and structural linkages at biological interfaces. Biophys. J. 68:2580-2587, 1995.

${ }^{26}$ Forman, J. R., and J. Clarke. Mechanical unfolding of proteins: insights into biology, structure and folding. Curr. Opin. Struct. Biol. 17:58-66, 2007.

${ }^{27}$ Frangos, J. A., L. V. McIntire, and S. G. Eskin. Shear stress induced stimulation of mammalian cell metabolism. Biotechnol. Bioeng. 32:1053-1060, 1988.

${ }^{28}$ Frye, S. R., A. Yee, S. G. Eskin, R. Guerra, X. Y. Cong, et al. CDNA microarray analysis of endothelial cells subjected to cyclic mechanical strain: importance of motion control. Physiol. Genomics 21:124-130, 2005.

${ }^{29}$ Galbraith, C. G., R. Skalak, and S. Chien. Shear stress induces spatial reorganization of the endothelial cell cytoskeleton. Cell Motil. Cytoskeleton 40:317-330, 1998.

${ }^{30}$ Galbraith, C. G., K. M. Yamada, and M. P. Sheetz. The relationship between force and focal complex development. J. Cell Biol. 159:695-705, 2002.

${ }^{31}$ Grimm, H. P., A. B. Verkhovsky, A. Mogilner, and J. J. Meister. Analysis of actin dynamics at the leading edge of crawling cells: implications for the shape of keratocyte lamellipodia. Eur. Biophys. J. 32:563-577, 2003.

${ }^{32}$ Gunnerson, K. N., Y. V. Pereverzev, and O. V. Prezhdo. Atomistic simulation combined with analytic theory to study the response of the P-selectin/PSGL-1 complex to an external force. J. Phys. Chem. B 113:2090-2100, 2009.

${ }^{33}$ Hammer, D. A., and S. M. Apte. Simulation of cell rolling and adhesion on surfaces in shear-flow. General results and analysis of selectin-mediated neutrophil adhesion. Biophys. J. 63:35-57, 1992.

${ }^{34}$ Hayakawa, K., H. Tatsumi, and M. Sokabe. Actin stress fibers transmit and focus force to activate mechanosensitive channels. J. Cell Sci. 121:496-503, 2008.

${ }^{35}$ Huang, J., J. Chen, S. E. Chesla, T. Yago, P. Mehta, et al. Quantifying the effects of molecular orientation and length on two-dimensional receptor-ligand binding kinetics. J. Biol. Chem. 279:44915-44923, 2004.

${ }^{36}$ Huang, J., V. I. Zarnitsyna, B. Y. Liu, L. J. Edwards, N. Jiang, et al. The kinetics of two-dimensional TCR and pMHC interactions determine T-cell responsiveness. Nature 464:932-936, 2010.

${ }^{37}$ Huisman, E. M., T. van Dillen, P. R. Onck, and E. Van der Giessen. Three-dimensional cross-linked F-actin networks: relation between network architecture and mechanical behavior. Phys. Rev. Lett. 99:208103, 2007.

${ }^{38}$ Icard-Arcizet, D., O. Cardoso, A. Richert, and S. Henon. Cell stiffening in response to external stress is correlated to actin recruitment. Biophys. J. 94:2906-2913, 2008.

${ }^{39}$ Inoue, Y., and T. Adachi. Coarse-grained Brownian ratchet model of membrane protrusion on cellular scale. Biomech. Model. Mechanobiol., 2010. doi:10.1007/s10237010-0250-6. 
${ }^{40}$ Interlandi, G., and W. Thomas. The catch bond mechanism between von Willebrand Factor and platelet surface receptors investigated by molecular dynamics simulations. Proteins 78:2506-2522, 2010.

${ }^{41}$ Kaplanski, G., C. Farnarier, O. Tissot, A. Pierres, A. M. Benoliel, et al. Granulocyte endothelium initial adhesion-analysis of transient binding events mediated by E-selectin in a laminar shear-flow. Biophys. J. 64:19221933, 1993.

${ }^{42}$ Kataoka, N., S. Ujita, and M. Sato. Effect of flow direction on the morphological responses of cultured bovine aortic endothelial cells. Med. Biol. Eng. Comput. 36:122-128, 1998.

${ }^{43}$ Keren, K., Z. Pincus, G. M. Allen, E. L. Barnhart, G. Marriott, et al. Mechanism of shape determination in motile cells. Nature 453:475-480, 2008.

${ }^{44}$ Kilian, K. A., B. Bugarija, B. T. Lahn, and M. Mrksich. Geometric cues for directing the differentiation of mesenchymal stem cells. Proc. Natl Acad. Sci. USA 107:48724877, 2010.

${ }^{45}$ Kim, T., W. Hwang, H. Lee, and R. D. Kamm. Computational analysis of viscoelastic properties of crosslinked actin networks. PLoS Comput. Biol. 5:e1000439, 2009.

${ }^{46}$ Klepeis, J. L., et al. Long-timescale molecular dynamics simulations of protein structure and function. Curr. Opin. Struct. Biol. 19(2):120-127, 2009.

${ }^{47}$ Krasik, E. F., K. E. Caputo, and D. A. Hammer. Adhesive dynamics simulation of neutrophil arrest with stochastic activation. Biophys. J. 95:1716-1728, 2008.

${ }^{48}$ Krasik, E. F., K. L. Yee, and D. A. Hammer. Adhesive dynamics simulation of neutrophil arrest with deterministic activation. Biophys. J. 91:1145-1155, 2006.

${ }^{49}$ Lacayo, C. I., Z. Pincus, M. M. VanDuijn, C. A. Wilson, D. A. Fletcher, et al. Emergence of large-scale cell morphology and movement from local actin filament growth dynamics. PLoS Biol. 5:2035-2052, 2007.

${ }^{50}$ LaMack, J. A., and M. H. Friedman. Individual and combined effects of shear stress magnitude and spatial gradient on endothelial cell gene expression. Am. J. Physiol. Heart Circ. Physiol. 293:H2853-H2859, 2007.

${ }^{51}$ Lauffenburger, D. A., and A. F. Horwitz. Cell migration: a physically integrated molecular process. Cell 84:359-369, 1996.

${ }^{52}$ Levesque, M. J., and R. M. Nerem. The elongation and orientation of cultured endothelial cells in response to shear stress. J. Biomech. Eng. 107:341-347, 1985.

${ }^{53}$ Levesque, M. J., R. M. Nerem, and E. A. Sprague. Vascular endothelial-cell proliferation in culture and the influence of flow. Biomaterials 11:702-707, 1990.

${ }^{54} \mathrm{Li}$, S., J. L. Guan, and S. Chien. Biochemistry and biomechanics of cell motility. Annu. Rev. Biomed. Eng. 7:105150, 2005.

${ }^{55}$ Liu, L., Y. Fang, Q. Huang, and J. Wu. A rigidity-enhanced antimicrobial activity: a case for linear cationic alpha-helical peptide HP(2-20) and its four analogues. PLoS One 6(1):e16441, 2011.

${ }^{56}$ Liu, Z., J. L. Tan, D. M. Cohen, M. T. Yang, N. J. Sniadecki, et al. Mechanical tugging force regulates the size of cell-cell junctions. Proc. Natl Acad. Sci. USA 107:99449949, 2010.

${ }^{57}$ Long, M., H. L. Goldsmith, D. F. J. Tees, and C. Zhu. Probabilistic modeling of shear-induced formation and breakage of doublets cross-linked by receptor-ligand bonds. Biophys. J. 76:1112-1128, 1999.
${ }^{58}$ Lou, J. Z., and C. Zhu. A structure-based sliding-rebinding mechanism for catch bonds. Biophys. J. 92:1471-1485, 2007.

${ }^{59}$ Lou, J. Z., and C. Zhu. Flow induces loop-to-beta-hairpin transition on the beta-switch of platelet glycoprotein $\mathrm{Ib}$ alpha. Proc. Natl Acad. Sci. USA 105:13847-13852, 2008.

${ }^{60}$ Lü, S. Q., and M. Long. Forced extension of P-selectin construct using steered molecular dynamics. Chin. Sci. Bull. 49:10-17, 2004.

${ }^{61}$ Lü, S. Q., Y. Zhang, and M. Long. Visualization of allostery in P-selectin lectin domain using MD simulations. PLoS One 5(12):e15417, 2010.

${ }^{62}$ Maree, A. F. M., A. Jilkine, A. Dawes, V. A. Grieneisen, and L. Edelstein-Keshet. Polarization and movement of keratocytes: a multiscale modelling approach. Bull. Math. Biol. 68:1169-1211, 2006.

${ }^{63}$ Matthews, B. D., D. R. Overby, R. Mannix, and D. E. Ingber. Cellular adaptation to mechanical stress: role of integrins, Rho, cytoskeletal tension and mechanosensitive ion channels. J. Cell Sci. 119:508-518, 2006.

${ }^{64}$ McBeath, R., D. M. Pirone, C. M. Nelson, K. Bhadriraju, and C. S. Chen. Cell shape, cytoskeletal tension, and RhoA regulate stem cell lineage commitment. Dev. Cell 6:483-495, 2004.

${ }^{65}$ Metaxa, E., H. Meng, S. R. Kaluvala, M. P. Szymanski, R. A. Paluch, et al. Nitric oxide-dependent stimulation of endothelial cell proliferation by sustained high flow. Am. J. Physiol. Heart Circ. Physiol. 295:H736-H742, 2008.

${ }^{66}$ Mogilner, A., and L. Edelstein-Keshet. Regulation of actin dynamics in rapidly moving cells: a quantitative analysis. Biophys. J. 83:1237-1258, 2002.

${ }^{67}$ Mogilner, A., and G. Oster. Cell motility driven by actin polymerization. Biophys. J. 71:3030-3045, 1996.

${ }^{68}$ Mogilner, A., and G. Oster. Force generation by actin polymerization II: the elastic ratchet and tethered filaments. Biophys. J. 84:1591-1605, 2003.

${ }^{69}$ Mogilner, A., and B. Rubinstein. The physics of filopodial protrusion. Biophys. J. 89:782-795, 2005.

${ }^{70}$ Morigi, M., C. Zoja, M. Figliuzzi, M. Foppolo, G. Micheletti, et al. Fluid shear-stress modulates surface expression of adhesion molecules by endothelial-cells. Blood 85:1696-1703, 1995.

${ }^{71}$ Nagel, T., N. Resnick, C. F. Dewey, and M. A. Gimbrone. Vascular endothelial cells respond to spatial gradients in fluid shear stress by enhanced activation of transcription factors. Thromb. Vasc. Biol. 19:1825-1834, 1999.

${ }^{72}$ Nakaya, M., M. Kitano, M. Masuda, and S. Nagata. Spationtemporal activation of Racl for engulfment of apoptotic cells. Proc. Natl Acad. Sci. USA 195:9198-9203, 2008.

${ }^{73}$ Nishio, K., Y. Ueki, N. Sakamoto, and M. Sato. Effect of initial orientation of vascular endothelial cells on activation of RhoGTPases induced by fluid shear stress. Cell. Mol. Bioeng. 2:160-168, 2011.

${ }^{74}$ Nomura, H., C. Ishikawa, T. Komatsuda, J. Ando, and A. Kamiya. A disk-type apparatus for applying fluid shearstress on cultured endothelial-cell. Biorheology 25:461-470, 1988.

${ }^{75}$ Paul, R., P. Heil, J. P. Spatz, and U. S. Schwarz. Propagation of mechanical stress through the actin cytoskeleton toward focal adhesions: model and experiment. Biophys. J. 94:1470-1482, 2008.

${ }^{76}$ Pawar, P., S. Jadhav, C. D. Eggleton, and K. Konstantopoulos. Roles of cell and microvillus deformation and 
receptor-ligand binding kinetics in cell rolling. Am. J. Physiol. Heart Circ. Physiol. 295:H1439-H1450, 2008.

${ }^{77}$ Peskin, C. S., G. M. Odell, and G. F. Oster. Cellular motions and thermal fluctuations: the Brownian ratchet. Biophys. J. 65:316-324, 1993.

${ }^{78}$ Phillips, J. C., R. Braun, W. Wang, J. Gumbart, E. Tajkhorshid, et al. Scalable molecular dynamics with NAMD. J. Comput. Chem. 26:1781-1802, 2005.

${ }^{79}$ Pollard, T. D., and G. G. Borisy. Cellular motility driven by assembly and disassembly of actin filaments. Cell 112:453-465, 2003.

${ }^{80}$ Poujade, M., E. Grasland-Mongrain, A. Hertzog, J. Jouanneau, P. Chavrier, et al. Collective migration of an epithelial monolayer in response to a model wound. Proc. Natl Acad. Sci. USA 104:15988-15993, 2007.

${ }^{81}$ Rubinstein, B., K. Jacobson, and A. Mogilner. Multiscale two-dimensional modeling of a motile simple-shaped cell. Multiscale Model. Sim. 3:413-439, 2005.

${ }^{82}$ Sakamoto, N., N. Saito, X. B. Han, T. Ohashi, and M. Sato. Effect of spatial gradient in fluid shear stress on morphological changes in endothelial cells in response to flow. Biochem. Biophys. Res. Commun. 395:264-269, 2010.

${ }^{83}$ Sasamoto, A., M. Nagino, S. Kobayashi, K. Naruse, Y. Nimura, et al. Mechanotransduction by integrin is essential for IL-6 secretion from endothelial cells in response to uniaxial continuous stretch. Am. J. Physiol. Cell Physiol. 288:C1012-C1022, 2005.

${ }^{84}$ Sato, M., K. Nagayama, N. Kataoka, M. Sasaki, and K. Hane. Local mechanical properties measured by atomic force microscopy for cultured bovine endothelial cells exposed to shear stress. J. Biomech. 33:127-135, 2000.

${ }^{85}$ Sawada, Y., M. Tamada, B. J. Dubin-Thaler, O. Cherniavskaya, R. Sakai, et al. Force sensing by mechanical extension of the Src family kinase substrate p130Cas. Cell 127:1015-1026, 2006.

${ }^{86}$ Shrader, C. D., H. G. Ressetar, J. Luo, E. V. Cilento, and F. D. Reilly. Acute stretch promotes endothelial cell proliferation in wounded healing mouse skin. Arch. Dermatol. Res. 300:495-504, 2008.

${ }^{87}$ Small, J. V., M. Herzog, and K. Anderson. Actin filament organization in the fish keratocyte lamellipodium. J. Cell Biol. 129:1275-1286, 1995.

${ }^{88}$ Sun, G. Y., Y. Zhang, B. Huo, and M. Long. Surfacebound selectin-ligand binding is regulated by carrier diffusion. Eur. Biophys. J. 38:701-711, 2009.

${ }^{89}$ Szymanski, M. P., E. Metaxa, H. Meng, and J. Kolega. Endothelial cell layer subjected to impinging flow mimicking the apex of an arterial bifurcation. Ann. Biomed. Eng. 36:1681-1689, 2008.

${ }^{90}$ Ter-Oganessian, N., B. Quinn, D. A. Pink, and A. Boulbitch. Active microrheology of networks composed of semiflexible polymers: computer simulation of magnetic tweezers. Phys. Rev. E 72:041510, 2005.

${ }^{91}$ Thery, M., A. Jimenez-Dalmaroni, V. Racine, M. Bornens, and F. Julicher. Experimental and theoretical study of mitotic spindle orientation. Nature 447:493-496, 2007.

${ }^{92}$ Thery, M., V. Racine, A. Pepin, M. Piel, Y. Chen, et al. The extracellular matrix guides the orientation of the cell division axis. Nat. Cell Biol. 7:947-953, 2005.

${ }^{93}$ Ueki, Y., N. Sakamoto, and M. Sato. Direct measurement of shear strain in adherent vascular endothelial cells exposed to fluid shear stress. Biochem. Biophys. Res. Commun. 394:94-99, 2010.

${ }^{94}$ Ueki, Y., N. Sakamoto, and M. Sato. Cyclic force applied to focal adhesions induces actin recruitment depending on the dynamic loading pattern. Open Biomed. Eng. J. 4:129134,2010

${ }^{95}$ Usami, S., H. H. Chen, Y. Zhao, S. Chien, and R. Skalak. Design and construction of a linear shear stress flow chamber. Ann. Biomed. Eng. 21:77-83, 1993.

${ }^{96}$ Verkhovsky, A. B., T. M. Svitkina, and G. G. Borisy. Network contraction model for cell translocation and retrograde flow. Biochem. Soc. Symp. 65:207-222, 1999.

${ }^{97}$ Vicente-Manzanares, M., X. Ma, R. S. Adelstein, and A. R. Horwitz. Non-muscle myosin II takes centre stage in cell adhesion and migration. Nat. Rev. Mol. Cell Biol. 10:778-790, 2009.

${ }^{98}$ Wang, G. M., and W. C. Sandberg. Complete all-atom hydrodynamics of protein unfolding in uniform flow. Nanotechnology 21:235101, 2010.

${ }^{99}$ Wang, N., and Z. Suo. Long-distance propagation of forces in a cell. Biochem. Biophys. Res. Commun. 328:1133-1138, 2005.

${ }^{100} \mathrm{Wu}$, J. H., Y. Fang, V. I. Zarnitsyna, T. P. Tolentino, M. L. Dustin, et al. A coupled diffusion-kinetics model for analysis of contact-area FRAP experiment. Biophys. J. 95:910-919, 2008.

${ }^{101}$ Wu, L., B. T. Xiao, X. L. Jia, Y. Zhang, S. Q. Lü, et al. Impact of carrier stiffness and microtopology on twodimensional kinetics of P-selectin and P-selectin glycoprotein ligand-1 (PSGL-1) interactions. J. Biol. Chem. 282:9846-9854, 2007.

${ }^{102}$ Wuang, S. C., B. Ladoux, and C. T. Lim. Probing the chemo-mechanical effects of an anti-cancer drug emodin on breast cancer cells. Mol. Cell Bioeng., 2011. doi: 10.1007/s12195-011-0163-1.

${ }^{103}$ Yago, T., J. Z. Lou, T. Wu, J. Yang, J. J. Miner, et al. Platelet glycoprotein Ibalpha forms catch bonds with human WT vWF but not with type 2B von Willebrand disease vWF. J. Clin. Invest. 118:3195-3207, 2008.

${ }^{104}$ Yam, P. T., C. A. Wilson, L. Ji, B. Hebert, E. L. Barnhart, et al. Actin-myosin network reorganization breaks symmetry at the cell rear to spontaneously initiate polarized cell motility. J. Cell Biol. 178:1207-1221, 2007.

${ }^{105} \mathrm{Yu}, \mathrm{Y}$., and J. Y. Shao. Simultaneous tether extraction contributes to neutrophil rolling stabilization: a model study. Biophys. J. 92:418-429, 2007.

${ }^{106}$ Zarbock, A., and K. Ley. Mechanisms and consequences of neutrophil interaction with the endothelium. Am. J. Pathol. 172:1-7, 2008.

${ }^{107}$ Zhang, F., W. D. Marcus, N. H. Goyal, P. Selvaraj, T. A. Springer, et al. Two-dimensional kinetics regulation of alpha(L)beta(2)-ICAM-1 interaction by conformational changes of the alpha(L)-inserted domain. J. Biol. Chem. 280:42207-42218, 2005.

${ }^{108}$ Zhao, Y. H., S. Chien, and S. Weinbaum. Dynamic contact forces on leukocyte microvilli and their penetration of the endothelial glycocalyx. Biophys. J. 80:1124-1140, 2001.

${ }^{109}$ Zou, X., Y. Liu, Z. Chen, G. I. Cardenas-Jiron, and K. Schulten. Flow-induced beta-hairpin folding of the glycoprotein Ibalpha beta-switch. Biophys. J. 99:11821191, 2010. 